between the halo and the nucleus. Dividing this by their velocity gives the time of halo emission (its zero date). The average difference between the zero dates of successive haloes was found to be 4.6 hours, this being the rotation period of the nucleus.

Whipple stresses quite rightly that this regularity of cometary behaviour is very strong evidence for the existence of a discrete central nucleus. In 1863 J. F. J. Schmidt analysed his own observations of Comet Donati and obtained a period of $4.3 \mathrm{~h}$. Unfortunately Schmidt did not take the next intuitive step and interpret his result in terms of a spinning concentrated nucleus.

The danger point for equatorial ejection occurs when the rotational surface velocity of the body is equal to its escape velocity. This occurs when the product of the period $T$ in hours and square root of the density $\rho$ in $g$ $\mathrm{cm}^{-3}$ is given by

$$
\mathrm{T} \rho^{\frac{1}{3}} \approx 1.32
$$

Hughes (J. Br. astr. Ass. 84, 272; 1974) found that the icy comet nucleus had a density of around $1.14 \mathrm{~g} \mathrm{~cm}^{-3}$ so it would be distinctly unstable if it were spinning with a period of less than 1.2 hours.

The period of around $4.6 \mathrm{~h}$ puts Comet Donati in the same ball park as asteroids (Psyche $4.3 \mathrm{~h}$, Vesta $5.3 \mathrm{~h}$, Eunomia $6.1 \mathrm{~h}$, Eros $5.3 \mathrm{~h}$, Ceres $9.1 \mathrm{~h})$. But it must be remembered that asymmetrical gas ejection can vary the cometary spin period. They can be spun-up until they become unstable and split, or they can spin down, becoming more stable and less prone to orbital changes produced by non-gravitational effects.

\section{Pion production in heavy-ion collisions}

from $P$. E. Hodgson

LAST year McNulty et al. (Phys. Rev. Lett. 38, 1519; 1977) measured the frequency of pion emission from the interactions of $280 \mathrm{MeV}$ per nucleon neon nuclei with nuclei in G5 nuclear emulsion and found that it was very much higher than the rate calculated by Bertsch from the independent particle model. This suggested that collective effects, perhaps associated with pion condensation, greatly enhance the frequency of pion production (see News and Views 268, 586; 1977).

It has now been found by Lindstrom et al. at Berkeley (Phys. Rev. Lett. 40, 93; 1978) that these measurements are probably in error, due to

\title{
ARC unit closes
}

\section{by Mary Lindley}

A SILver jubilee and a death knell were marked simultaneously on 25 April with a botanical reunion at Wye College in Kent. After 25 years of major contributions to plant science, the Agricultural Research Council's Unit of Plant Growth Substances and Systemic Fungicides is being closed on the retirement of its director $R$. $L$. Wain. He is departing to the peaceful pastures of an emeritus professorship at the University of Kent, and his staff, friends and colleagues past and present paid tribute to him with a symposium.

The unit has been in the forefront of research into the properties and application of naturally occurring plant substances. As Wain reminded the visitors, those substances have included abscisic acid, so named for its role in leaf abscission; xanthoxin, a hormone inhibitor and 'a Wye product through and through'; and the fungicide wyerone. Wain's own special genius has been for chemical synthesis, according to L. Fowden (Rothamsted Experimental Station). He described Wain as a great manipulator of the carbon-halogen bond, who has produced compounds that have since assumed great value as, for example, herbicides and specific growth antagonists. The value of such achievements was endorsed by Sir William Henderson (Agricultural Research Council, London) whose search of the council's archives had revealed that the funds voted for the unit's first year of operation in 1953 amounted to a munificent $£ 4,650$.

The staff of the unit will be taking their expertise to four of the council's institutes-Rothamsted Experimental Station, Long Ashton Research

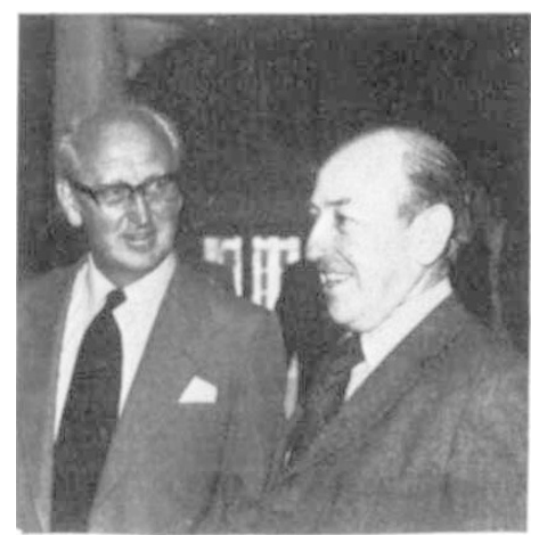

Professor R. L. Wain (left) and Sir William Henderson.

Station, the Weed Research Organization and the Letcombe Laboratory, all concerned in some way with the promotion or inhibition of plant growth. Past and future interests of the members of the unit were reflected in the contributions to the symposium. In particular there was the affection of T. A. Mansfield (University of Lancaster) for stomatathose remarkable sense organs, which, in contrast to popular belief, do not merely open by day and close by night. He would like to see plant hormones used to manipulate them and thus influence photosynthetic efficiency. F. Wightman (Carlton University, Ottawa) is adding new vigour to plant studies with the news that phenylacetic acid seems to be one of a new group of naturally occurring plant growth promoters. Whatever future work may reveal about the control and manipulation of plant growth, those from Wye will inevitably be in the thick of it.

Mary Lindley is an Assistant Editor of Nature. the misidentification of proton tracks as pion tracks. It is not easy to distinguish between the tracks of relativistic protons and pions in nuclear emulsions, because both have ionisation close to the minimum value. McNulty $e t$ al. used multiple scattering measurements to do this, and Lindstrom et al. conclude that these must have been in error.

In their work, Lindstrom et al. examined a similar stack of emulsion exposed to neon nuclei of about the same energy, and looked for pions coming to rest in the emulsion. This is a much more definite measurement because the tracks of pions and protons can easily be distinguished near the ends of their ranges. In an area of emulsion that should, if the previously reported production rate were accurate, contain the tracks of about 50 stopping pions they did not find any. This shows that the rate of pion production is less than 0.06 of that found by McNulty et al. and is thus not inconsistent with the predictions of the independent particle model.

P. E. Hodgson is a Lecturer in Nuclear Physics at the University of Oxford. 